\title{
Optimal Design Parameters for Wireless Power Transfer by Resonance Magnetic
}

\author{
Olutola Jonah, Student Member, IEEE, Stavros V. Georgakopoulos, Senior Member, IEEE, and \\ Manos M. Tentzeris, Fellow, IEEE
}

\begin{abstract}
The wireless power transfer (WPT) efficiency of systems based on strongly coupled magnetic resonance (SCMR) depends on the $Q$-factor of the system elements, which is a function of the geometrical parameters. This letter analytically derives the equations that can be used to design optimal loop and helical elements for SCMR systems. Also, for loops and helices, a global maximum condition is derived in order to design SCMR systems with maximal efficiency.
\end{abstract}

Index Terms-Geometric parameters limits, magnetic resonance, wireless sensor.

\section{INTRODUCTION}

$\mathbf{N}$ UMEROUS wireless power transfer methods have been proposed and studied in the past for various applications. Specifically, wireless power transfer has been achieved using near-field coupling in several applications such as RFID tags, telemetry, and implanted medical devices [1], [2]. In addition, certain inductive coupling techniques have been reported to exhibit high power transfer efficiencies (on the order of $90 \%$ ) for very short distances $(1-3 \mathrm{~cm})[3]$. However, the efficiency of such techniques drops drastically for longer distance since it decays as $1 / r^{6}$ [4], [5].

This letter focuses on the design of wireless powering systems based on the strongly coupled magnetic resonance (SCMR) method. The SCMR method is a novel nonradiative wireless midrange power transfer method $(10-300 \mathrm{~cm})$ that has been recently developed [6]-[11]. In fact, the SCMR method has achieved $40 \%$ wireless power transfer efficiency in air for a distance of $2 \mathrm{~m}$ for a single receiver [6]. Also, SCMR technique has been used to simultaneously power multiple receivers in air and has achieved $60 \%$ at a distance of $2 \mathrm{~m}$ [7]. Previous work has shown that SCMR provides wireless power transfer efficiencies that are significantly larger than efficiencies of conventional inductive coupling methods [6], [7], [12]. In order for SCMR to achieve high efficiency, it requires that the

Manuscript received June 19, 2012; revised August 12, 2012 and September 20, 2012; accepted October 16, 2012. Date of publication November 15, 2012; date of current version December 07, 2012. This work was supported in part by Florida International University under the Dissertation Year Fellowship.

O. Jonah is with the Department of Electrical and Computer Engineering, Florida International University, Miami, FL 33172 USA (e-mail: ojona001@fiu. edu).

S. V. Georgakopoulos is with the Engineering Center, Telecommunications and Information Technology Institute, Florida International University, Miami, FL 33174 USA (e-mail: georgako@fiu.edu).

M. M. Tentzeris is with the Georgia Electronic Design Center, School of Electrical and Computer Engineering, Georgia Institute of Technology, Atlanta, GA 30332 USA (e-mail: etentze@ece.gatech.edu).

Digital Object Identifier 10.1109/LAWP.2012.2228459

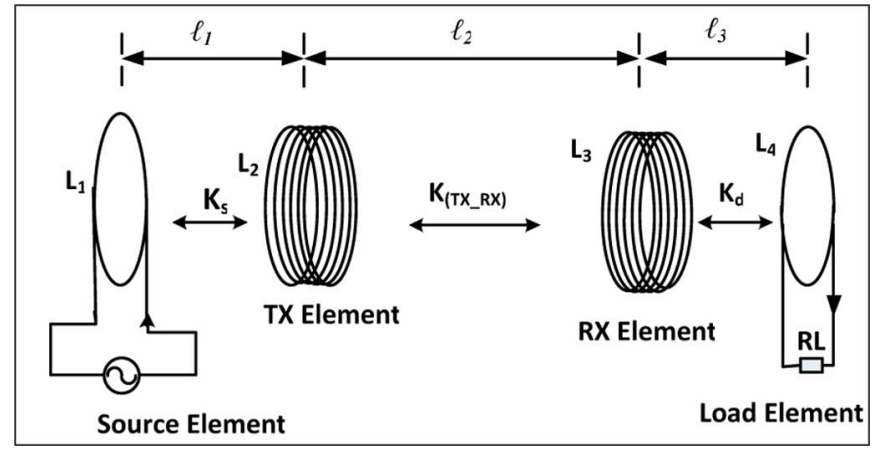

Fig. 1. Schematic of an SCMR power transfer system in air

transmitting and receiving elements (typically loops or coils) are designed so that they resonate at the desired operational frequency that must coincide with the frequency of where the elements exhibit maximum $Q$-factor.

This letter analytically derives equations that must be satisfied by the geometrical parameters of loops or helices in order to guarantee optimal efficiency for SCMR systems.

\section{Wireless POWERING With SCMR}

SCMR systems use resonant transmitters and receivers that are strongly coupled. Strongly coupled systems are able to transfer energy efficiently because resonant objects exchange energy efficiently versus nonresonant objects that only interact weakly. A standard SCMR system consists of four elements (typically four loops or two loops and two coils) as shown in Fig. 1.

The source element is connected to the power source, and it is inductively coupled to the TX element. The TX element exhibits a resonant frequency that coincides with the frequency, where its $Q$-factor is naturally maximum. Similarly, the RX exhibits a resonant frequency that coincides with the frequency where its $Q$-factor is naturally maximum. Furthermore, the load element is terminated to a load. For our analysis, we assume that the entire system operates in air. Also, SCMR requires that the TX and RX elements are resonant at the same frequency in order to achieve efficient wireless power transfer.

\section{OPTIMAL SCMR BASED ON LOOPS AND HELICES}

In this section, we will derive the guidelines for designing SCMR systems that uses helices as TX and RX elements. The TX and RX elements can be equivalently represented by a series $R L C$ circuit. Helices are often preferred as TX and RX SCMR elements because they exhibit both distributed inductance and 
capacitance, and therefore they can be designed to self-tune to a desired resonant frequency $f_{\mathrm{r}}$ without the need of external capacitors. Also, external capacitors have losses, which in practice can reduce the $Q$-factor of the TX and RX elements and in turn decrease the efficiency of SCMR systems. Based on the equivalent $R L C$ circuit of an SCMR system, its resonant frequency $f_{\mathrm{r}}$ can be calculated by the following equation:

$$
f_{r}=\frac{1}{2 \pi \sqrt{L C}}
$$

The resonant frequency $f_{\mathrm{r}}$ is also the operational frequency for the SCMR wireless powering system. The $Q$-factor of a resonant $R L C$ circuit is given by

$$
Q=\frac{\omega_{\mathrm{r}} L}{R}=\frac{2 \pi f_{\mathrm{r}} L}{R}
$$

Therefore, the $Q$-factor of a resonant helix (i.e., self resonant) can be written as

$$
Q=\frac{2 \pi f_{\mathrm{r}} L_{\mathrm{helix}}}{R_{\mathrm{ohm}}+R_{\mathrm{rad}}}
$$

where $L, R_{\text {rad }}$, and $R_{\text {ohm }}$ are the self-inductance, radiation resistance, and ohmic resistance of the helix, which is for a short helix or solenoid $(2 r>h)$ given by [4], [13]-[15]

$$
\begin{aligned}
L_{\text {helix }} & =\mu_{o} r N^{2}\left[\ln \left(\frac{8 r}{r_{\mathrm{c}}}\right)-2\right] \\
R_{\mathrm{rad}} & =\left(\frac{\pi}{6}\right) \eta_{o} N^{2}\left(\frac{2 \pi f_{\mathrm{r}} r}{c}\right)^{4} \\
R_{\text {ohm(helix })} & =\left(\sqrt{\mu_{o} \rho \pi f_{\mathrm{r}}}\right) \frac{N r}{r_{\mathrm{c}}}
\end{aligned}
$$

where $\mu$ is the permeability of free space, $\rho$ is the helix's material resistivity, $r$ is the radius of the helix, $r_{\mathrm{c}}$ is the cross sectional wire radius, $N$ is the number of turns, $f$ is the frequency, $\eta_{o}$ is the impedance of free space, $c$ is the speed of light, and $h$ is the height of the helix. It should also be noted that (3)-(6) are valid only when $r<\lambda / 6 \pi$ [3].

SCMR requires that both $\mathrm{RX}$ and TX helices also exhibit maximum $Q$-factor at their resonant frequency $f_{\mathrm{r}}$ in order to achieve maximum wireless power efficiency. This can also be seen by the equation for describing the efficiency of an SCMR system derived in at it operation frequency $f_{\mathrm{r}}[16]$ as follows:

$$
\eta\left(f_{\mathrm{r}}\right)=\frac{k_{\left(\mathrm{TX} \_\mathrm{RX}\right)}^{2}\left(f_{r}\right) Q_{\mathrm{TX}}\left(f_{\mathrm{r}}\right) Q_{\mathrm{RX}}\left(f_{\mathrm{r}}\right)}{1+k_{\left(\mathrm{TX} \_\mathrm{RX}\right)}^{2}\left(f_{\mathrm{r}}\right) Q_{\mathrm{TX}}\left(f_{\mathrm{r}}\right) Q_{\mathrm{RX}}\left(f_{\mathrm{r}}\right)}
$$

where $K_{\mathrm{TX} \_\mathrm{RX}}$ is the mutual coupling between the RX and TX helices, whereas $Q_{\mathrm{TX}}$ and $Q_{\mathrm{RX}}$ are the $Q$-factors of the RX and TX helices, respectively. If the TX and RX helices are identical their $Q$-factors are equal i.e., $Q_{\mathrm{TX}}=Q_{\mathrm{RX}}=Q$; therefore, (7) can be written as

$$
\eta\left(f_{\mathrm{r}}\right)=\frac{k_{\left(\mathrm{TX} \_\mathrm{RX}\right)}^{2}(f) Q_{\mathrm{TX}}^{2}\left(f_{\mathrm{r}}\right)}{1+k_{\left(\mathrm{TX} \_\mathrm{RX}\right)}^{2}\left(f_{\mathrm{r}}\right) Q_{\mathrm{TX}}^{2}\left(f_{\mathrm{r}}\right)} .
$$

Equation (8) shows that in order to maximize the efficiency of an SMCR system, the operation frequency $f_{\mathrm{r}}$ must be equal to the frequency $f_{\max }$, where the $Q$-factor is maximum. In what follows, the maximum $Q$-factor of a resonant helix is derived. The $Q$-factor of a resonant helix can be expressed in terms of its geometrical parameters using (3)-(6) as

$$
Q\left(f_{\mathrm{r}}, r, r_{\mathrm{c}}, N\right)=\frac{2 \pi f_{\mathrm{r}} \mu_{0} r N^{2} \ln \left(\frac{8 r}{r_{\mathrm{c}}-2}\right)}{\left(\frac{\mu_{0} \rho \pi f_{\mathrm{r}} r^{2} N}{r_{\mathrm{c}}^{2}}\right)^{1 / 2}+20 \pi^{2} N^{2}\left(\frac{2 \pi f_{\mathrm{r}} r}{c}\right)^{4}} .
$$

The maximum $Q$-factor $Q_{\max }$ and the frequency $f_{\max }$, where $Q_{\max }$ occurs, can be derived from (9) using calculus as

$$
\begin{aligned}
& f_{\max }\left(r, r_{\mathrm{c}}, N\right) \\
& \quad=\frac{c^{8 / 7} \mu^{1 / 7} \rho^{1 / 7}}{4 \cdot 15^{2 / 7} N^{2 / 7} r_{\mathrm{c}}^{2 / 7} \pi^{11 / 7} r^{6 / 7}} \\
& Q_{\max }\left(r, r_{\mathrm{c}}, N\right) \\
& \quad=\frac{2 \pi f_{\max } \mu_{0} r N^{2} \ln \left(\frac{8 r}{r_{\mathrm{c}}-2}\right)}{\left(\frac{\mu_{0} \rho \pi r^{2} f_{\max } N}{r_{\mathrm{c}}^{2}}\right)^{1 / 2}+20 \pi^{2} N^{2}\left(\frac{2 \pi f_{\max } r}{c}\right)^{4}} .
\end{aligned}
$$

Based on the above discussion, an SCMR system requires that

$$
f_{\mathrm{r}}=f_{\max }
$$

which can be written based on (10) as

$$
f_{\mathrm{r}}\left(r, r_{\mathrm{c}}, N\right)=\frac{c^{8 / 7} \mu^{1 / 7} \rho^{1 / 7}}{4 \cdot 15^{2 / 7} N^{2 / 7} r_{\mathrm{c}}^{2 / 7} \pi^{11 / 7} r^{6 / 7}} .
$$

Therefore, (13) shows that the geometrical parameters of a helix can be appropriately chosen so that the helix has maximum $Q$-factor at a chosen frequency $f_{\mathrm{r}}$. For example, if the parameters $f_{\mathrm{r}}, r_{\mathrm{c}}, N$, and $\rho$ are specified by a designer, (13) can be solved for the radius of the maximum $Q$-factor, $r_{\max }$, as follows:

$$
r_{\max }=\left[\frac{c^{8 / 7} \mu^{1 / 7} \rho^{1 / 7}}{4 \cdot 15^{2 / 7} r_{\mathrm{c}}^{2 / 7} N^{2 / 7} \pi^{11 / 7} f_{\mathrm{r}}}\right]^{7 / 6} .
$$

Similar equations to (10), (11), and (13) have been presented in [17] in order to obtain the local maximum for the $Q$-factor.

Next, the helices are analyzed using (10), (11), and (14) to study the behavior of the maximum $Q$-factor $Q_{\max }$ versus the electrical length of the helix $\left(C_{\operatorname{dev}} / \lambda_{Q_{\max }}\right)$ at $f_{\max }$, which can be written as

$$
\frac{C_{\mathrm{dev}}}{\lambda_{\max }}=\frac{2 \pi r_{\max }}{\lambda_{\max }}=\frac{2 \pi r_{\max } f_{\max }}{c}
$$

where $L_{\mathrm{dev}}$ is the length of the helix (device), and $\lambda_{\max }$ is the wavelength corresponding to $f_{\max }$ given by (10). Specifically, optimum SCMR helices with $N=6$ are designed in the frequency range $100 \mathrm{kHz} \leq f \leq 5 \mathrm{GHz}$ for four values of the cross-sectional radius, $r_{\mathrm{c}}=0.01,0.1,1.0$, and $10 \mathrm{~mm}$. The material of the helices is assumed copper, and for each pair of $f_{\max }$ and $r_{\mathrm{c}}$, the optimum $r$ is calculated by (14). Then, $Q_{\max }$ from (11) is plotted in Fig. 2 versus the electrical length of the helices $\left(C_{\operatorname{dev}} / \lambda_{Q_{\max }}\right)$, which is calculated by (15). Specifically, Fig. 2 illustrates that for each pair of $f_{\max }$ and $r_{\mathrm{c}}$, there is an $r_{\text {max }}$ that provides the global maximum for the $Q$-factor, $Q_{G \text { max }}$. 


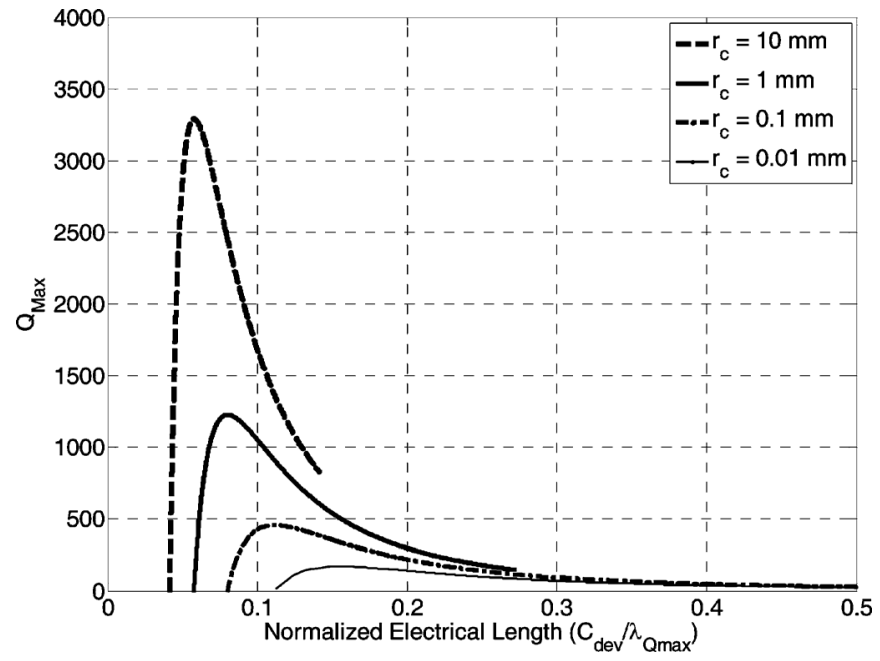

Fig. 2. $Q_{\max }$ versus electrical length of the helix.

In what follows, the global maximum $Q$-factor of the helix, $Q_{G \max }$, is formulated. First, the local maximum $Q$-factor, $Q_{L \max }$, is derived by substituting (10) into (11)

$$
\begin{aligned}
& Q_{L \max }= \\
& \frac{2 \cdot 3^{6 / 7} r_{\mathrm{c}}^{6 / 7} c^{8 / 7} \mu^{8 / 7} N^{6 / 7} \rho^{1 / 7}\left[\ln \left(\frac{8 r}{r_{\mathrm{c}}}\right)-2\right]}{5^{1 / 7} \pi^{2 / 7} r^{3 / 7}\left[c^{4 / 7} \mu^{4 / 7} \rho^{4 / 7}+6 r_{\mathrm{c}}^{1 / 7} N^{1 / 7} r^{3 / 7} \sqrt{\frac{c^{8 / 7} \mu^{8 / 7} \rho^{8 / 7}}{r_{\mathrm{c}}^{2 / 7} N^{2 / 7} r^{6 / 7}}}\right]} .
\end{aligned}
$$

Using calculus again, we can find out that the global maximum for the $Q$-factor occurs when

$$
\frac{r_{(G \max )}}{r_{\mathrm{c}}}=\frac{e^{13 / 3}}{8} \approx 9.52
$$

This is an important finding, which shows that the ratio between the helix radius $r$ and the cross-sectional radius $r_{c}$ must be approximately 9.52 in order to achieve the maximum $Q$-factor. This ratio is also independent of frequency and material.

Also, by substituting (17) into (16), we can write the global maximum for the $Q$-factor as

$$
\begin{aligned}
& Q_{G \max }= \\
& \frac{28 \cdot 2^{2 / 7} r_{\mathrm{c}}^{3 / 7} c^{8 / 7} \mu^{8 / 7} N^{6 / 7} \rho^{1 / 7}}{15^{1 / 7} e^{13 / 7} \pi^{2 / 7}\left[c^{4 / 7} \mu^{4 / 7} \rho^{4 / 7}+6 r_{\mathrm{c}}^{4 / 7} N^{1 / 7} \sqrt{\frac{c^{8 / 7} \mu^{8 / 7} \rho^{8 / 7}}{r_{\mathrm{c}}^{8 / 7} N^{2 / 7}}}\right]} .
\end{aligned}
$$

Therefore, if a helix is designed to operate at the global maximum $Q$-factor, it will yield the maximum possible wireless efficiency for the corresponding SCMR system. In order to verify the global maximum design of (17), we assume that an arbitrary ratio of $r / r_{c}=t$ and solve (13) and (17) to obtain the $r$ and $r_{\mathrm{c}}$ given the number of turns, $N$, and the desired frequency of operation, $f_{o}$

$$
\begin{aligned}
r_{\mathrm{c}_{\max }} & =\frac{c \mu^{1 / 8} \rho^{1 / 8}}{2 \cdot 2^{3 / 4} \cdot 15^{1 / 4} N^{1 / 4} f_{o}^{7 / 8} \pi^{11 / 8} t^{3 / 4}} \\
r_{\max } & =\frac{c \mu^{1 / 8} \rho^{1 / 8} t^{1 / 4}}{2 \cdot 2^{3 / 4} \cdot 15^{1 / 4} N^{1 / 4} f_{o}^{7 / 8} \pi^{11 / 8}} .
\end{aligned}
$$

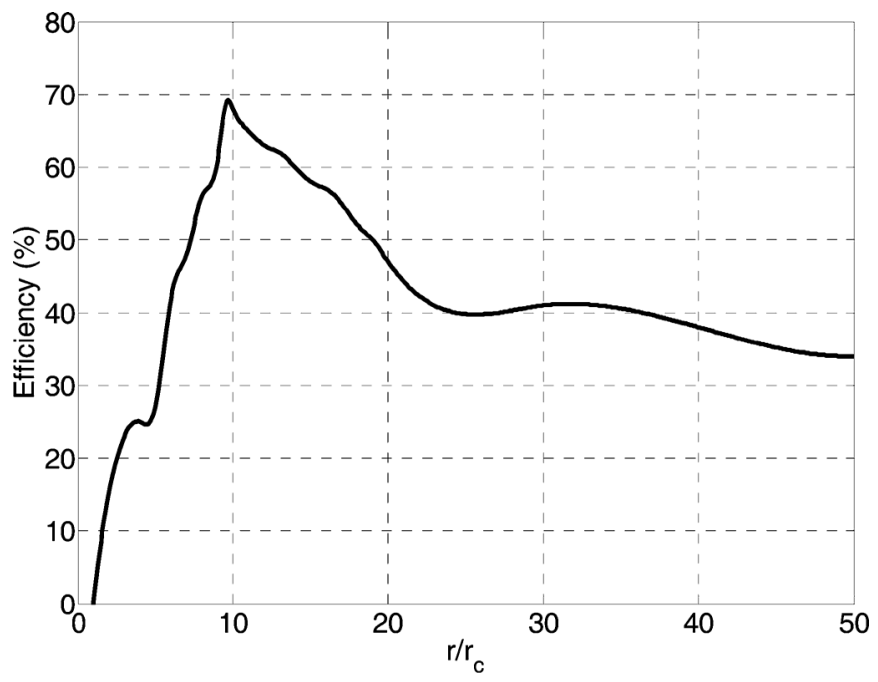

Fig. 3. Efficiency of SCMR systems with different $r / r_{\mathrm{c}}$ ratios.

Based on (19) and (20), SCMR systems were designed and simulated in Ansoft HFSS for different ratios $r / r_{\mathrm{c}}(2 \leq t \leq 50)$ and assuming the number of turns, $N=5$; distances, $l_{1}=$ $l_{3}=2 \mathrm{~cm}, l_{2}=10 \mathrm{~cm}$ (see Fig. 1); and operational frequency, $f_{o}=46.5 \mathrm{MHz}$. The efficiency of these designs is compared in Fig. 3 . The results clearly illustrate that the maximum efficiency is achieved for a ratio of $t=9.52$ that matches our derived global maximum condition of (17).

\section{Optimized Spacing IN SCMR BASEd ON Helices}

In this section, we will examine the guidelines for designing helical TX and RX elements of SCMR wireless powering systems. An SCMR system based on helices will not be optimal unless the spacing $s$ is picked so that the helices exhibit the appropriate capacitance in order to resonate at the desired operating frequency of the system. In [18], it was also shown that the spacing $s$ of an SCMR helix is an important parameter that should be carefully picked to ensure optimal wireless power transfer efficiency. The capacitance formula for closely wound helix was presented in [19] as follows:

$$
C_{\mathrm{t}}=\frac{2 \pi^{2} r \varepsilon_{0}}{\ln \left[\frac{s}{2 r_{\mathrm{c}}}+\sqrt{\left(\frac{s}{2 r_{\mathrm{c}}}\right)^{2}-1}\right]}
$$

where $r$ is the radius of the helix, $r_{\mathrm{c}}$ is the cross-sectional wire radius, $\varepsilon_{0}$ is the permittivity of free space, $s$ is the spacing between adjacent turns of the helix, $C_{\mathrm{t}}$ is the total distributed capacitance of the helix, and $t$ is the thickness of the insulation coating.

The capacitance formula of (21) is valid when $s / 2 r_{\mathrm{c}} \leq 2$ and $t \ll s-2 r_{\mathrm{c}}$. In order to resonate the helix at a desired frequency $f$, the spacing between two adjacent turns, $s$, can be adjusted to provide the required capacitance calculated from (1) as

$$
C_{\mathrm{t}}=\frac{1}{4 \pi^{2} f^{2} L_{\mathrm{helix}}} .
$$

Then, (21) can be solved for the spacing $s$ as follows:

$$
s=\frac{\left[e^{\left(4 \pi^{4} r^{2} \varepsilon_{0}^{2} / C_{\mathrm{t}}^{2}\right)}+1\right] r_{\mathrm{c}}}{e^{\left(2 \pi^{2} r \varepsilon_{0} / C_{\mathrm{t}}\right)}} .
$$


Equation (23) is valid when $s / 2 r_{\mathrm{c}} \leq 2$ and $t \ll s-2 r_{\mathrm{c}}$. Therefore, the spacing $s$ can be adjusted using (23) independently from the other geometrical parameters to achieve the necessary capacitance and without affecting the frequency where a short helix or solenoid $(2 r>h)$ exhibits maximum $Q$-factor since (13) shows that the $f_{\max }$ does not depend on $s$.

\section{CONCLUSION}

This letter analytically examines the optimal design of SCMR elements. Specifically, helices are analyzed. We analytically derived equations that can be used to design SCMR systems. In addition, our formulations proved that the global maximum $Q$-factor of a helix is achieved when the ratio between the radius $r$ and the cross-sectional radius $r_{\mathrm{c}}$ of the helix is approximately equal to 9.52 . This important finding can be used to design maximally efficient SCMR wireless powering systems that use coils or helices as transmitting and receiving elements.

\section{REFERENCES}

[1] K. Finkenzeller, RFID Handbook: Fundamentals and Applications in Contactless Smart Cards and Identification, 2nd ed. New York: Wiley, 2003, pp. 65-112.

[2] P. V. Nikitin, K. V. S. Rao, and S. Lazar, "An overview of near field UHF RFID," in Proc. RFID IEEE Int. Conf., Mar. 2007, pp. 167-174.

[3] G. Vandevoorde and R. Puers, "Wireless energy transfer for standalone systems: A comparison between low and high energy applicability," Sensors Actuators A, Phys., vol. 92, no. 1-3, pp. 305-311, Aug. 2001.

[4] C. A. Balanis, Antenna Theory: Analysis and Design. Hoboken, NJ: Wiley, 2005, ch. 5.

[5] S. J. Mazlouman, A. Mahanfar, and B. Kaminska, "Mid-range wireless energy transfer using inductive resonance for wireless sensors," in Proc. IEEE Int. Conf. Comput. Design, 2009, pp. 517-522.
[6] A. Kurs, A. Karalis, R. Moffatt, J. D. Joannopoulos, P. Fisher, and M. Soljacic, "Wireless energy transfer via strongly coupled magnetic resonances," Science, vol. 317, pp. 83-85, 2007.

[7] A. Kurs, A. Karalis, R. Moffatt, and M. Soljacic, "Simultaneous midrange power transfer to multiple devices," Appl. Phys. Lett., vol. 96, pp. 044102-044102, 2010.

[8] A. Karalis, J. D. Joannopoulos, and M. Soljacic, "Efficient wireless non-radiative mid-range energy transfer," Ann. Phys., vol. 323, pp. 34-48, Jan. 2008.

[9] D. Joannopoulos, A. Karalis, and M. Soljacic, "Wireless non-radiative energy transfer," US Patent 20070222542, Sep. 2007.

[10] N. P. Cook, P. Meier, L. Sieber, M. Secall, and H. Widmer, "Wireless energy apparatus and method," US Patent 20080211320, Sep. 2008.

[11] B. L. Cannon, J. F. Hoburg, D. D. Stancil, and S. C. Goldstein, "Magnetic resonant coupling as a potential means for wireless power transfer to multiple small receivers," IEEE Trans. Power Electron., vol. 24, no. 7, pp. 1819-1825, Jul. 2009.

[12] O. Jonah and S. V. Georgakopoulos, "Wireless power transmission to sensors embedded in concrete via magnetic resonance," in Proc. 12th Annu. IEEE Wireless Microw. Technol. Conf., Apr. 2011, pp. 1-6.

[13] R. Lundin, "A handbook formula for the inductance of a single-layer circular coil," Proc. IEEE, vol. 73, no. 9, pp. 1428-1429, Sep. 1985.

[14] D. Fang, Handbook of Electrical Calculations. Jinan, China: Shandong Sci. Technol. Press, 1994.

[15] J. L. Volakis, Antenna Engineering Handbook, 4th ed. New York: McGraw-Hill, 2007, ch. 5.

[16] A. K. RamRakhyani, S. Mirabbasi, and C. Mu, "Design and optimization of resonance-based efficient wireless power delivery systems for biomedical implants," IEEE Trans. Biomed. Circuits Syst., vol. 5, no. 1, pp. 48-63, Feb. 2011.

[17] A. Karalis, A. Kurs, R. Moffat, D. Joannopoulos, P. H. Fisher, and M. Soljacic, "Wireless energy transfer," US Patent 20110193419A1, Aug. 2011.

[18] O. Jonah and S. V. Georgakopoulos, "Optimal helices for wireless power transfer via magnetic resonance," in Proc. 13th Annu. IEEE WAMICON, Apr. 15-17, 2012, pp. 1-4.

[19] G. Grandi, M. K. Kazimierczuk, A. Massarini, and U. Reggiani, "Stray capacitances of single-layer solenoid air-core inductors," IEEE Trans. Ind. Appl., vol. 35, no. 5, pp. 1162-1168, Sep.-Oct. 1999. 